\title{
Stress effects on working memory, explicit memory, and implicit memory for neutral and emotional stimuli in healthy men
}

\author{
Mathias Luethi ${ }^{1}$, Beat Meier ${ }^{1 *}$ and Carmen Sandi ${ }^{2}$ \\ 1 Department of Psychology, University of Bern, Bern, Switzerland \\ 2 Laboratory of Behavioral Genetics, Brain Mind Institute, Ecole Polytechnique Fédérale de Lausanne, Switzerland
}

\section{Edited by:}

Sonia Lupien, McGill University,

Canada

Reviewed by:

Tony W. Buchanan, Saint Louis

University, USA

Dominique J. F. de Quervain, University

of Zurich, Switzerland

${ }^{*}$ Correspondence:

Beat Meier, Department of Psychology, University of Bern, Muesmattstr. 45

CH-3000 Bern 9, Switzerland.

e-mail: beat.meier@psy.unibe.ch
Stress is a strong modulator of memory function. However, memory is not a unitary process and stress seems to exert different effects depending on the memory type under study. Here, we explored the impact of social stress on different aspects of human memory, including tests for explicit memory and working memory (for neutral materials), as well as implicit memory (perceptual priming, contextual priming and classical conditioning for emotional stimuli). A total of 35 young adult male students were randomly assigned to either the stress or the control group, with stress being induced by the Trier Social Stress Test (TSST). Salivary cortisol levels were assessed repeatedly throughout the experiment to validate stress effects. The results support previous evidence indicating complex effects of stress on different types of memory: A pronounced working memory deficit was associated with exposure to stress. No performance differences between groups of stressed and unstressed subjects were observed in verbal explicit memory (but note that learning and recall took place within $1 \mathrm{~h}$ and immediately following stress) or in implicit memory for neutral stimuli. Stress enhanced classical conditioning for negative but not positive stimuli. In addition, stress improved spatial explicit memory. These results reinforce the view that acute stress can be highly disruptive for working memory processing. They provide new evidence for the facilitating effects of stress on implicit memory for negative emotional materials. Our findings are discussed with respect to their potential relevance for psychiatric disorders, such as post traumatic stress disorder.

Keywords: stress, cortisol, implicit memory, working memory, conditioning, emotional stimuli

\section{INTRODUCTION}

There is substantial evidence that stress and enhanced glucocorticoid levels can have complex influences on memory performance, with both negative and positive consequences (Lupien and Lepage, 2001; Lupien et al., 2007; Sandi and Pinelo-Nava, 2007; Wolf, 2003). Critical brain areas for cognition and emotion - such as the hippocampus and the amygdala in rodents, and the hippocampus and frontal lobe in humans - contain a high density of glucocorticoid receptors (de Kloet et al., 1999; Lupien and McEwen, 1997).

In rodents, converging evidence suggests that stress effects on hippocampus- and prefrontal cortex-dependent memory follow an inverted $\mathrm{U}$-shaped function, with moderate stress levels facilitating, while high levels impairing, memory function (Cordero and Sandi, 1998; Del Arco et al., 2007; Sandi and Pinelo-Nava, 2007; Sandi et al., 1997; Selden et al., 1990). Glucocorticoids seem to play a key role in these stress effects, since an inverted U-shaped function has also been reported for the relationship between glucocorticoid levels and memory and plasticity (Abrari et al., 2008; Joëls, 2006; Sandi and Rose, 1997). Conversely, hippocampus-independent memory is frequently facilitated by stress (Sandi and Pinelo-Nava, 2007; Shors, 2004, 2006), and this facilitation seems to be dependent on glucocorticoids (Shors, 2001; Shors and Beylin, 2003).

In humans, explicit memory and working memory formation have been shown to be frequently impaired after corticosteroid administration and as a result of psychological stress (Kirschbaum et al., 1996; Lupien et al., 1999; Newcomer et al., 1994, 1999; Oei et al., 2006; Payne et al., 2007). However, there are also examples in which a potentation of memory was observed after corticosterone treatment (Buchanan and Lovallo, 2001; Putman et al., 2004) or when psychological stress was applied before (Payne et al., 2007) or after (Smeets et al., 2008) training. For implicit memory, the small number of studies that have addressed this issue suggest that performance is unaffected by stress and elevated cortisol levels (Kirschbaum et al., 1996; Lupien et al., 1997).

One of the key issues addressed in recent years has been the potentially different susceptibility of different memory phases (i.e., acquisition, consolidation, retrieval) to the effects of acute stress and increased cortisol levels (Roozendaal et al., 2002; Smeets et al., 2008). Evidence from studies on explicit memory suggest that retrieval processes are particularly susceptible to the adverse effects of acute stress and increased cortisol, while consolidation processes could be in fact potentiated by both stress and glucocorticoids (Beckner et al., 2006; de Quervain et al., 2000; Het et al., 2005; Lupien and Schramek, 2006; Roozendaal et al., 2002).

Another key issue has been to ascertain whether the emotional modulation of memory formation - in which amygdala activation has been critically involved (Cahill, 2003) - occurs for both positive and negative materials. Findings in rodents suggest that 
the enhancing effect of glucocorticoids on memory consolidation depend on emotional arousal (e.g., Roozendaal et al., 2006). In humans, a memory bias towards negative stimuli is often described in patients whose condition is associated with elevated levels of stress hormones (Colombel, 2007; Elzinga and Bremner, 2002; Moradi et al., 2000; Rinck and Becker, 2005; Watkins et al., 2000). In a recent study (Abercrombie et al., 2006), high cortisol output during a social stressor was related to memory facilitation in subjects who reported high stress-related negative affect, and this relation was especially prominent for recall of unpleasant information. Other studies also found impaired recall for negative and positive words, but no effect for neutral words (Kuhlmann et al., 2005; Tops et al., 2003). In accordance with findings in rodents, stress induced facilitation of the implicit learning of emotionally negative information was also found in humans (Gidron et al., 2002). Similarly, Jackson et al. (2006) report enhanced fear conditioning after stress exposure in men, but not in women. This effect was associated with elevated cortisol levels. Interestingly, the impairing effects of glucocorticoids on memory retrieval seem to also depend on emotional arousal (e.g., Roozendaal et al., 2006) both for positive and negative information (de Quervain et al., 2007).

The aim of this study was to assess the effects of stress and cortisol on a comprehensive variety of memory tasks in male human subjects, including tests for explicit memory (for neutral materials), working memory, and implicit memory (perceptual and contextual priming and classical conditioning for emotional stimuli), with a particular focus on different types of implicit memory. Stress was induced in half of the sample through the Trier social stress test (TSST; Kirschbaum et al., 1993). Explicit memory was studied through a standardized explicit memory test (LGT-3; Bäumler, 1974). Working memory was assessed with the reading span task (Daneman and Carpenter, 1980). For implicit memory, a perceptual priming task, a contextual priming task, and a conditioning task were used. The priming tasks consisted of neutral materials, while the conditioning task included both positive and negative stimuli. To validate the effects of social stress, salivary cortisol was sampled repeatedly throughout the experiment. Based on previous findings, we expected a negative effect of stress on explicit memory and working memory. In contrast, we did not expect stress effects on implicit memory for non-emotional materials, but hypothesized facilitative effect of stress on implicit memory for the condition with emotionally congruent materials.

\section{MATERIALS AND METHODS SUBJECTS AND DESIGN}

Thirty-five healthy male volunteers, aged $23.4 \pm 2.9$ years $(M \pm S D)$, range 20-34 years, participated in this study. They were randomly assigned to either the stress $(n=19)$ or the control group $(n=16)$. Groups did not differ with respect to age or education level.

All subjects were informed that the experiment might be partly unpleasant and that they were free to leave at any time. They signed a consent form prior to testing. All subjects were medication-free. At least $1 \mathrm{~h}$ prior to testing ( $1 \frac{1 / 2}{\mathrm{~h}}$ prior to the first saliva sample), participants refrained from exercise, smoking (smoking $>10$ cigarettes/ day was an exclusion criteria), eating, or drinking alcoholic beverages or low $\mathrm{pH}$ soft drinks. Each subject completed a questionnaire to confirm good health and compliance with dietary instructions.

\section{SALIVA SAMPLING AND FREE CORTISOL ANALYSIS}

Saliva was collected using Salivette (Sarstedt, Sevelen, Switzerland) collection devices. Saliva samples were taken at the end of a relaxation phase to assess baseline cortisol levels (sample 1); and $15 \mathrm{~min}$ after stress cessation, or $15 \mathrm{~min}$ after the beginning of memory testing in controls to assess peak levels or comparison levels, respectively (sample 2). Sample 3 was taken at the end of the memory testing phase. Samples were stored at $-30^{\circ} \mathrm{C}$ until analysis. Cortisol concentration was measured using the Spectria Cortisol radioimmunoassay RIA commercial kit (Orion Diagnostica, Espoo, Finland).

\section{GENERAL PROCEDURE}

Subjects were tested individually in the second half of the day between 11 a.m.-1 p.m. $(n=9), 2-4$ p.m. $(n=14)$ or $4-6$ p.m. $(n=12)$. Test sessions lasted $1 \frac{1 / 2}{2}-2 \mathrm{~h}$ and consisted of a relaxation phase (25 $\mathrm{min}$ ), exposure to a social stressor ( $25 \mathrm{~min}$; only for the stress group) and a memory testing phase $(1 \mathrm{~h})$. After completion of a post-experimental questionnaire, subjects were debriefed. Controls started memory testing immediately after the relaxation phase. Table 1 shows an overview of the order of the specific tasks and activities.

\section{Relaxation phase}

After arrival at the laboratory $(t 0)$, subjects rested while completing a socio demographic-questionnaire. They were instructed to take as much time as they needed to answer the questions. In case subjects did not manage to complete the questionnaire within $30 \mathrm{~min}$, they were told to stop ( $t 30)$, see Table 1.

\section{Stress exposure}

Subjects in the stress group were exposed to the Trier Social Stress Test (TSST; Kirschbaum et al., 1993) at t30 (see Table 1). Subjects were told that the TSST procedure is about simulating a job interview. The TSST mainly consists of a stress anticipation period, a free speech and mental arithmetic task to be performed in front of an audience. Members of the "audience" (these were colleagues of the first author) were introduced as being trained in observing nonverbal behaviour. Subjects had to stand close to a microphone and a video camera. They were told that their performance would be videotaped for subsequent analysis. A powerful light source was directed towards the subjects and they saw themselves on a monitor screen connected to the video camera. During performance subjects were given negative feedback about their level of achievement by the audience.

\section{MEMORY TESTS}

\section{Explicit memory}

Explicit memory was assessed using two sub-tests of a standardized memory test (LGT-3; Bäumler, 1974). The first test was a verbal memory test. Subjects were presented with a list of 20 German and Turkish words (stress group: t60/control group: t30). They were instructed to learn both the German words and the Turkish translations and were given 1 min to study the list. The second test was a spatial memory test. Subjects were instructed to learn a route on a map $(t 61 / t 31)$. They were also given $1 \mathrm{~min}$ for study. Free recall and recognition were tested at $1113-116$ in the stress group, and t83-86 in the control group, respectively (see Table 1). 
Table 1 | Ordering of tasks and activities.

\begin{tabular}{|c|c|c|c|}
\hline \multirow[t]{2}{*}{ Activity } & \multirow[t]{2}{*}{ Duration } & \multicolumn{2}{|c|}{ Cumulative time } \\
\hline & & Stress group & Control group \\
\hline Stress exposure & $25 \mathrm{~min}$ & t30-55 & \\
\hline \multicolumn{4}{|l|}{ Explicit memory study } \\
\hline Classical conditioning study & $12 \mathrm{~min}$ & $t 62-74$ & t32-44 \\
\hline Perceptual priming study & $4 \mathrm{~min}$ & $t 74-78$ & $t 44-48$ \\
\hline Contextual priming task & $12 \mathrm{~min}$ & t78-90 & $t 48-60$ \\
\hline Classical conditioning evaluation & $2 \mathrm{~min}$ & t90-92 & $t 60-62$ \\
\hline Perceptual priming test & $7 \mathrm{~min}$ & t92-99 & t62-69 \\
\hline \multicolumn{4}{|l|}{ END OF MEMORY TESTING } \\
\hline \multicolumn{4}{|l|}{ Contextual priming } \\
\hline Awareness test & $1 \mathrm{~min}$ & $t 116$ & $t 86$ \\
\hline \multicolumn{4}{|l|}{ Classical conditioning } \\
\hline Awareness questionnaire & $1 \mathrm{~min}$ & $t>116$ & $t>86$ \\
\hline Debriefing & $5 \mathrm{~min}$ & $t>116$ & $t>86$ \\
\hline
\end{tabular}

\section{Working memory}

Working memory was assessed with a modified version of the reading span task (Daneman and Carpenter, 1980). Subjects were instructed to read aloud a set of sentences and to indicate whether they were meaningful. In addition, they were also instructed to memorize the last word of each sentence and to recall these last words in the correct order at the end of a trial. Trials consisted of sets of two, three, four, five or six sentences. There were five trials for each set size. After a practice trial, the test trials started with sets of two sentences. If the subject was able to recall all the words in at least one of the five trials, five trials with three sentences were presented, etc. If subjects failed to recall all the words of a given set size in at least one trial of a given set size, the task was stopped. Reading span was defined as the size of the largest set in which all words were correctly recalled in at least three of the five trials. If subjects correctly recalled all words of two sets at a given set size, the reading span was scored as the size of this set minus 0.5 (cf. Daneman and Carpenter, 1980). Working memory was assessed at $199-113$ in the stress group and t69-83 in the control group (see Table 1).

\section{Perceptual priming}

Perceptual priming was assessed with a fragmented pictures test (cf. Meier, 2001; Meier et al., 2009). A total of 100 line drawings of common objects from materials of Snodgrass and Vanderwart (1980) were used. They were presented in black against a white background on a computer screen. Four groups of 25 line-drawings were composed such that each group had the same baseline completion rate. During study two sets of 25 line drawings were presented and participants were instructed to perform a simple decision task (study phase; $t 74-78 / t 44-48$ ). Each display was presented for $1 \mathrm{~s}$, followed by a blank screen, during which subjects responded. After a filled delay, subjects were given a fragmented pictures test ( $t 92-99$ / t62-69; see Table 1). A total of 100 pictures were presented in random order. Half of them were previously shown and half were new objects. Subjects were instructed to name each object. They were also informed that objects would be difficult to identify because they were shown in fragmented form. First, the most fragmented version of an object was shown for $3 \mathrm{~s}$. If the subject was unable to name the object correctly, the same object was presented in a less fragmented version. This procedure was repeated until the object was named correctly. If an object was identified correctly, the next object appeared on the screen, again in its most fragmented version first. The level of picture fragmentation, at which an object was identified, was recorded. For each object, six fragmentation levels were used with objects being complete in the last version. The four lists of items were counterbalanced across conditions. Priming was calculated as the difference between the fragmentation level at which old (i.e., previously seen) and new drawings were identified.

\section{Contextual priming}

Contextual priming was assessed with a paradigm from Chun and Jiang (1998; t78-90/t48-60, see Table 1). Materials consisted of a total of 90 search displays presented on a computer screen. Each display featured 12 coloured items presented in a small square $(4 \times 2.5 \mathrm{~cm})$ against a grey background. There were equal numbers of red, green, blue and yellow items in each display. Each display consisted of 11 distracters and 1 target item that appeared anywhere within a grid of $8 \times 6$ locations. Distracter items were $\mathrm{L}-$ letters, which were rotated 
through $0,90,180$ or 270 degrees. The target item was a T, which was rotated through 90 degrees either in clockwise or counter-clockwise direction. In each block, five "new" displays and five "old" displays were shown in randomised order. Each type of "old" display was presented once in a block, and eight times across the experiment. Within each "old" display, the spatial and colour configuration of the items was the same. Hence, over the repetitions, the visual context predicted the location of the target item as "old" displays differed only with respect to the orientation of the target item. "New" displays differed with respect to both the spatial and colour configurations of the items. Each trial began with the presentation of a fixation cross and after a 500-ms delay, a visual search display appeared. Subjects were instructed to indicate as quickly and accurately as possible the direction of the T-base. The visual search display disappeared if one of the response keys was pressed, or after a maximum of $6 \mathrm{~s}$. The next trial was initiated $1000 \mathrm{~ms}$ after response. Feedback was given for incorrect responses. Priming was assessed as differential speed-up in RTs for old vs. new items across blocks. For analysis Blocks 1 and 2, Blocks 3 and 4, Blocks 5 and 6, and Blocks 7 and 8 were summarized as Epoch 1 to 4 in order to enhance statistical power. At $t 116 / t 86$, subjects were asked whether they were aware of repeated presentation of "old" displays (see Table 1). Additionally, a recognition test featuring "old" and "new" displays as well as displays not used in the experiment was conducted to test for explicit learning of stimulus configurations.

\section{Classical conditioning}

To assess conditioning for emotional material a paradigm by Olson and Fazio (2001) was used. Subjects were told that the task was about "video surveillance", and that several hundred pictures would be presented on a computer screen $(t 62-74 / t 32-44$, see Table 1$)$. They were instructed to hit a response key as quickly as possible when a target appeared. Target events were defined as a name or a picture of a Pokemon cartoon character. They could appear either alone or paired with other photographs or other words. Subjects were told to focus on monitoring for targets and not to get distracted by the other items. These filler items consisted of other Pokemon figures or names, blank screens, and neutrally valenced words and pictures (see Olson and Fazio, 2001). A total of five blocks, each consisting of 86 trials, was administered. Eight pairs of conditioned stimuli (CS) and unconditioned stimuli (UCS) were presented in each block. These pairs consisted of a Pokemon character and a positively valenced word (e.g., "excellent”) or picture (e.g., a puppy) and another Pokemon cartoon character paired with a negative word (e.g., "terrible") or picture (e.g., a cockroach). Following the procedure of Olson and Fazio, the pokemon characters Shelder and Metapod were used as CSs. Pokemon character and US were counterbalanced across conditions. After the conditioning phase, subjects were asked to complete a picture evaluation task ( 190 or $t 60$, respectively). They were told that one purpose of this task was to control for interference effects of some filler items. Thirty photographs and Pokemon characters, including the "positive" CS (CS pos) and the "negative" CS (CS neg) were presented at a rapid pace on the computer screen. Subjects were asked to evaluate the pictures on a scale ranging from extremely negative $(-4)$ to extremely positive $(+4)$ as quickly as possible. A conditioning effect was defined as a more positive rating of CS pos relative to CS neg characters. CS-US covariation awareness was controlled using a funnelled multiple-choice questionnaire at the end of the testing phase $(t 116 / t 86$, see Table 1$)$.

\section{STATISTICS}

Results are expressed as mean $(M) \pm$ standard deviation (SD). Mean differences between the two groups were assessed by means of Student $t$-tests. Significance was considered at $p<0.05$.

\section{RESULTS SALIVARY CORTISOL LEVELS}

Salivary cortisol levels did not differ between the stress and control group at baseline $(M=10.0 \mathrm{nmol} / \mathrm{L}, S D=3.9 \mathrm{vs.} M=8.6 \mathrm{nmol} / \mathrm{L}$, $S D=4.7)$, but they were elevated in the stress group $15 \mathrm{~min}$ after the beginning of the testing phase $[M=23.2 \mathrm{nmol} / \mathrm{L}, S D=13.6 \mathrm{vs}$. $M=9.6 \mathrm{nmol} / \mathrm{L}, S D=3.2 ; t(20.5)=4.2, p<0.001]$ and at the end of the testing phase $[M=15.6 \mathrm{nmol} / \mathrm{L}, S D=7.2 \mathrm{vs} . M=10.6 \mathrm{nmol} / \mathrm{L}$, $S D=3.2 ; t(26)=2.7, p=0.01]$. Salivary cortisol levels rose significantly in response to the TSST $[t(18)=-4.1, p=0.001]$, whereas controls showed no difference in cortisol levels between samples 1 and 2. The mean cortisol increase (sample 2-sample 1) in the experimental group was $13.1 \mathrm{nmol} / \mathrm{L}$. The increase in cortisol concentration could not be calculated in one control subjects due to an insufficient amount of saliva.

Cortisol has a pronounced diurnal pattern. Accordingly, the baseline cortisol concentration tended to differ between the different testing times in the afternoon $[F(2,32)=2.7, p=0.08]$. However, the baseline cortisol concentration was not associated with the increase in cortisol concentration, nor did the cortisol response differ between subjects with low vs. high cortisol concentrations at baseline (groups based on median cortisol level $=7.58 \mathrm{nmol} / \mathrm{L}$ ). These results validate the efficacy of the TSST in inducing stress. There was a clear-cut endocrine response to the psychological stressor which was not significantly influenced by the cortisol concentration at baseline.

\section{MEMORY TESTS}

Descriptive statistics for all memory tests are presented in Table 2. For all statistical analyses an alpha-level of 0.05 was used. Due to an experimenter error, working memory data of one control and two stressed subjects and verbal explicit memory data of one control subject had to be excluded from analysis.

\section{Explicit memory}

Measures of the verbal explicit memory test were indistinguishable between the stress and the control group. Subjects in the stress group achieved higher scores in the spatial memory test $[t(33)=2.1, p=0.046]$.

\section{Working memory}

Subjects exposed to the TSST had a lower reading span $[t(28.5)=-2.1, p=0.046]$ as well as significantly lower total correct scores $[t(30)=-2.4, p=0.023]$ relative to controls. The results suggest a stress-induced working memory impairment.

\section{Perceptual priming}

Lower mean fragmentation levels at which objects were correctly identified, indicate higher levels of object fragmentation 
Table 2 | Descriptive statistics indicating means and standard deviations $(M \pm S D)$ of memory test results in the experimental and the control group. Significant differences are indicated in italics $(p<0.05)$.

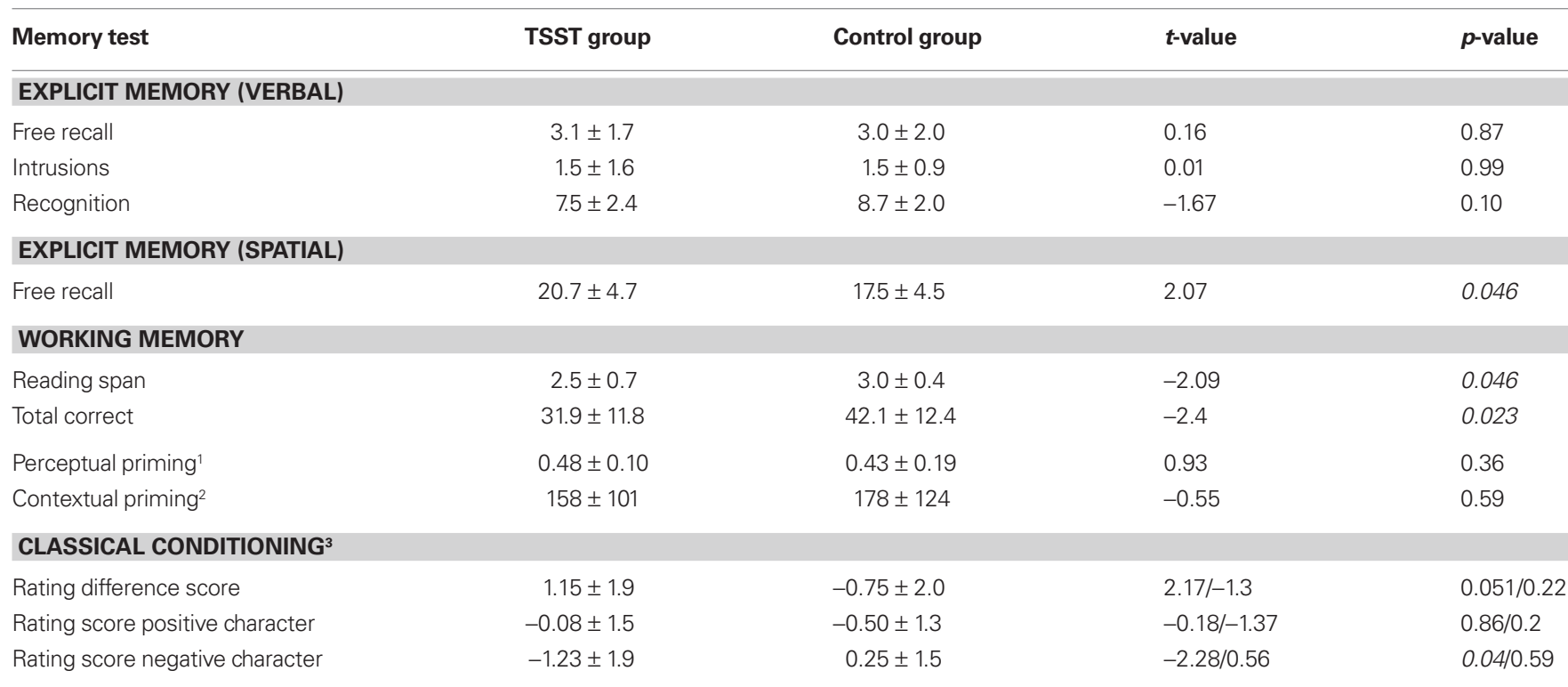

'Difference in fragmentation level between old and new items.

${ }^{2} R T$ difference between new and old displays in the second half of the experiment.

${ }^{3}$ One-sample test, test value $=0$.

and consequently, better object recognition. A paired Student $t$-test revealed a significant difference in mean fragmentation levels between previously seen objects (i.e., old) and new objects $[t(34)=18.0, p<0.001]$, with significant facilitation for old items. Priming scores were calculated by subtracting the level of object fragmentation of old and new items. The amount of priming did not differ between stressed and unstressed subjects.

\section{Contextual priming}

To measure learning effects, reaction times of correct responses were analysed (mean response accuracy was $98 \%$ ). A repeated measures ANOVA revealed a significant interaction between "Display Type" (old vs. new displays) and "Epoch" (epoch 1-4) $(F[3,102]=6.6$, $p<0.001$ ), indicating context-dependent learning. Following the convention by Chun and Jiang (1998), the magnitude of contextual learning was defined as the difference in performance between old and new display conditions over the latter half of the experimental sessions (Epochs 3 and 4). Repeated measures ANOVAs showed a main effect of "Display Type" $(F[1,33]=78.5, p<0.001)$, indicating a significant priming effect but no main effect of stress and no interaction between "Display Type" and "Group" (stress vs. control group), suggesting no effect of stress on contextual priming.

In the post-experimental questionnaire, 15 out of the 35 subjects reported that they thought that display repetitions had occurred. However, subjects performed at chance in discriminating old displays from new displays and those never shown in a recognition test: Hit rates were indistinguishable from false positive rates in the total sample and in a subsample of subjects who reported that they had noticed the repetitions. Subjects who reported being aware of repetitions did not differ from the remaining subjects in a display recognition test: No between group differences were found in hit rates, nor in false positive rates. In addition, the magnitude of contextual cueing was indistinguishable between the aware and the unaware group. Thus, subjects who reported being aware of display repetitions were not excluded from the analysis. The results indicate that memory for context was implicit, but nevertheless facilitated search performance. The magnitude of this effect did not differ between groups of stressed and unstressed subjects, implying a lack of stress effects on implicit contextual learning.

\section{Classical conditioning}

25 subjects reported that they were not aware of anything unusual during the presentation of the two CS Pokemon characters, even when presented with the names of these characters. The remaining 10 subjects ( 6 stressed, 4 controls) were excluded from data analysis due to CS-US covariation awareness of at least one of the two types of critical pairings. Difference scores were calculated between CS pos and CS neg ratings. These scores differed significantly between the TSST and the control group $[t(23)=2.4, p=0.023]$. According to Student $t$-tests, difference scores were marginally different from zero in the group of subjects exposed to the TSST $[t(12)=2.2$, $p=0.051]$, but not in the control group. A further analysis revealed that conditioning effects in stressed subjects were due to the negative ratings of the negative Pokemon character (CS neg). The ratings for this character differed significantly from zero in subjects exposed to the TSST $[t(12)=-2.3, p=0.041]$, but not in the control subjects (see Figure 1). Also, CS neg ratings differed between stressed and control subjects [ $t(23)=-2.1, p=0.047]$. In contrast, ratings of the positive Pokemon character (CS pos) did not differ from zero in either condition, nor between the two experimental groups (see Figure 1). Hence, the conditioning effects observed in stressed subjects were due to valence specific stimulus processing, with a bias towards negative stimuli. The specific Pokemon character that was paired with the positive US (i.e., the CS pos) 


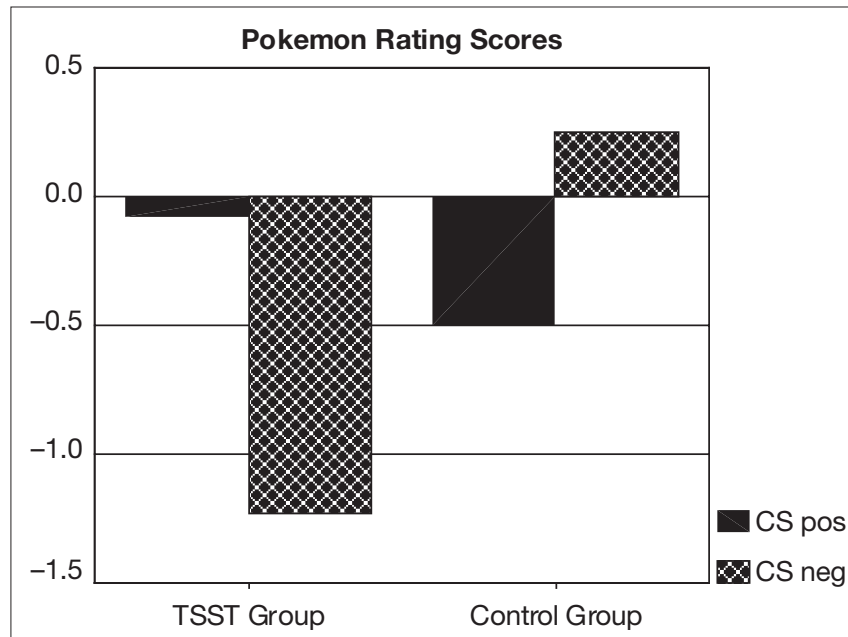

FIGURE 1 | Pokemon rating scores for CS pos and CS neg in stressed and control subjects.

or negative US (i.e., the CS neg) had no effect. Neither the rating difference score, nor the ratings for the CS pos or the CS neg were different for the two Pokemon characters.

\section{DISCUSSION}

The goal of this study was to investigate the effects of stress and cortisol on a comprehensive variety of memory tasks, including tests for explicit memory, working memory, and implicit memory, and with special emphasis on these latter ones. One advantage of our experiment was that all these different memory tasks were assessed in the same experimental procedure, which allowed us to compare the impact of stress on different domains of memory. Our results support the view that working memory is sensitive to disruption under our experimental conditions (note that both learning and recall of explicit learning took place within the hour following stress application). In contrast verbal episodic memory was not affected by stress, while spatial episodic memory was enhanced. We found no effect of stress on implicit learning for neutral stimuli. However, our results showed an enhancement of implicit memory for negative, but not positive, emotional stimuli. This latter result is particularly relevant since it suggests a mood congruency effect of stress in conditioning.

Explicit verbal memory was previously documented to be negatively affected by stress and high cortisol levels (Lupien et al., 2005; Sauro et al., 2003; Wolf, 2006), with strong evidence indicating that retrieval processes are particularly vulnerable (de Quervain et al., 2000, 2003; Het et al., 2005; Roozendaal, 2002). Stress and glucocorticoids may have opposing effects on explicit memory consolidation and retrieval, with enhancing effects on consolidation and impairing effects on retrieval (e.g. Beckner et al., 2006; Roozendaal, 2002; Sandi and Pinelo-Nava, 2007; Smeets et al., 2008). In this context, a potential role of reconsolidation on the facilitating effects of stress on consolidation has been discussed (Lupien and Schramek, 2006). Under our experimental conditions, verbal explicit memory for emotionally neutral materials was not affected by stress. This could be related to the fact that learning and retrieval occurred under the same stressful conditions: Potentially enhancing effects of stress on consolidation might have balanced out potentially impairing effects on retrieval. It could be speculated that a stress-induced enhancement of consolidation processes during the retention interval may account for the performance increase in the stress group for the spatial explicit memory test. However, consolidation mechanisms are believed to occur over hours (Morris, 2006), even days, and therefore the short delay taking place in our study for the different tasks between training and testing might have not be sufficient for potential stress effects on consolidation to take place. Alternatively, the stress and cortisol levels induced in our study may have not been strong enough to disrupt memory retrieval.

Our data support previous evidence indicating a clear impairment of verbal working memory after stress (Lupien et al., 1999; Oei et al., 2006; Robinson et al., 2008; Schoofs et al., 2008). We found that exposure to a social stressor impaired working memory performance. This is in line with the notion that stress affects abilities that require conscious, effortful information processing and therefore reduces cognitive efficiency. However, within the framework of a general adaptation to stress, it might be indeed an adaptive process compensated for by increased automatic processing efficiency in the case of important stimuli, such as potentially negative and threatening events (de Kloet et al., 1999). These processes might be mediated by the release of stress hormones such as cortisol. However, such a mechanism could be potentially maladaptive in conditions of chronic stress and could, therefore, be involved in the development and maintenance of psychiatric conditions such as depression, post traumatic stress disorder (PTSD) and phobias (Elzinga and Bremner, 2002; Wolf, 2008).

The main finding of this paper concerns the differential stress effects observed for different implicit memory tests. Perceptual priming and contextual priming both involving neutral stimuli were not affected by stress. These findings are in agreement with previous work reporting an absence of stress effects in implicit memory (Kirschbaum et al., 1996; Lupien et al., 1997). However, the contextual cueing task which is thought to be dependent upon hippocampal functioning (Chun and Phelps, 1999; Greene et al., 2007) was also unaffected by the stress manipulation. As in the case of explicit verbal memory, an explanation may be that both study and test phase were administered under stressful conditions and that any facilitating effect of stress at encoding may have been levelled out by interfering effects at retrieval. Typically, stress effects are most pronounced when retrieval in a stress situation is required for information that has been acquired under non-stressful conditions (de Quervain et al., 2000, 2003; Het et al., 2005; Roozendaal, 2002).

For the conditioning task, we expected a stress induced modulation of performance in line with the mood congruency hypothesis (Colombel, 2007). It was reasoned that due to the aversive characteristics of stress, this effect might be valence-specific, with a bias towards negative stimuli (Bishop, 2007; Wolf, 2008). Conditioning effects were observed in stressed but not control subjects. This finding is consistent with studies in animals reporting enhanced conditioning after stress when using aversive paradigms, such as fear conditioning (Conrad et al., 1999; Cordero et al., 2003a,b; Sandi et al., 2001; Shors, 2001,2006), as well as fear conditioning in healthy men (Jackson et al., 2006; Zorawski et al., 2006). In our study, conditioning effects in stressed subjects were due to the negative rating of the CS neg. In contrast, the rating for the CS pos did not differ 
from zero in stressed subjects. This suggests that stress can enhance implicit memory for emotional stimuli in a valence-specific manner, with a bias towards negative materials. The processing bias towards negative stimuli might be due to a bias in attention, learning or in the willingness to report negative attitudes (Bishop, 2007; Rinck and Becker, 2005). As in explicit memory, stress or cortisol enhanced implicit memory is likely to be due to enhanced memory consolidation (Zorawski et al., 2006). As mentioned above, enhanced automatic cognitive processing during stress could be thought of as both an adaptive process and a potentially maladaptive mechanism. The former holds true from a cognitive resources viewpoint, that is, when conscious and effortful processing of information is decreased, and more automatic information processing is increased. However, from a clinical viewpoint, the same mechanism could be potentially maladaptive, with negative stimuli appearing to be the ones that are processed more efficiently during times of stress. In line with Jackson et al. (2006) who found similar conditioning effects, we suggest that the enhancing effects of stress on the formation of implicit negative attitudes provide a model of pathological emotional reactions, such as those found in PTSD.

Interestingly, elevated levels of glucocorticoids have also been discussed as protective agents with regard to the development and symptomatology of anxiety disorders, such as PTSD and phobias (Aerni et al., 2004; de Quervain, 2006, 2008; Soravia et al., 2006). However, these studies used explicit, self report measures of anxiety or anxiety related memories. It is possible that glucocorticoids impair the retrieval of negative or anxiety-related explicit episodic memories, but still enhance implicit learning of negative stimuli, as suggested by our study and others, who describe enhanced implicit memory for trauma-related materials (McNally, 1997). Indeed, memories of PTSD patients are often characterized by vivid, dreamlike flashbacks, yet patients find it difficult to retrieve specific, autobiographical memories from their past (McNally, 1997).

Hence, stress exposure and glucocorticoids could be thought of as both a protective mechanism as well as a risk factor in the development and maintenance of PTSD. In fact, one could speculate that the discrepancy between enhanced implicit and impaired explicit processing of anxiety related stimuli might itself be a risk factor in PTSD. In accordance with this view, a mnemonic model of PTSD has been suggested, with a focus on the current memory of a negative event, as opposed to the event itself (Rubin et al., 2008). Psychotherapeutic interventions in PTSD are also in line with such a discrepancy between implicit and explicit memories in PTSD patients, as they often focus on the patient's memory for the event and involve re-experiencing the traumatic event (e.g. Ehlers and Clark, 2008). It is plausible that such interventions reduce discrepancies between implicit and explicit memories, thereby reducing PTSD symptoms.

Another explanation for the protective role of glucocorticoids in PTSD could be that the same type of memory is affected by glucocorticoids in opposite ways depending on the memory phase exposed. Based on the findings that glucocorticoids enhance memory consolidation, it can be assumed that elevated glucocorticoid levels at the time of an aversive experience may contribute to the formation (and strength) of traumatic memories. Indeed, a study in critically ill patients found that the number of traumatic memories from the intensive care unit correlated positively with the dose of cortisol acutely administered to patients undergoing cardiac surgery (Schelling, 2008). On the other hand, prolonged administration of stress-equivalent doses of cortisol during intensive care treatment was found to reduce the risk for later PTSD (Schelling, 2008). After initial consolidation of traumatic experiences (which is likely to be enhanced by glucocorticoids), glucocorticoid levels may play a crucial role in controlling the amount of retrieved traumatic memories later on. Specifically, by the known reducing effects of glucocorticoids on memory retrieval, these hormones may partly interrupt the vicious cycle of retrieving, re-experiencing and reconsolidating aversive memories, thereby preventing a further cementation of the aversive memory trace (de Quervain, 2008). Studies showing that the preventive effects of glucocorticoid administration are also observed when the treatment starts at the time of the traumatic event (Schelling et al., 2004; Weis et al., 2006) indicate that such an inhibitory effect of glucocorticoids on memory retrieval may prevail over their potentially enhancing effect on initial consolidation.

In summary, the degree of stress and the enhanced cortisol levels induced by our experimental conditions were sufficient to impair working memory, enhance spatial episodic memory, and to facilitate classical conditioning for aversive stimuli. In contrast, they did not affect performance in verbal explicit memory tasks, or in implicit learning tasks that involved neutral or positive stimuli. Given the different brain regions which are hypothesized to play a major role in orchestrating each of these memory tasks, our results suggest that stress may reduce the efficiency of prefrontal cortex processing (working memory) and yet facilitate the efficiency of amygdala processing (aversive conditioning). At the same time, stress did not seem to negatively affect hippocampal processing, as required for explicit memory and implicit memory tasks. However, this is still somewhat speculative as, in the present study, we did not counterbalance the order of the memory tests. Therefore, it may be that variations of stress levels across the different memory tests, as well as variations of stress effects across the different processes involved in each memory test (i.e., encoding, consolidation and retrieval), also contributed to the differential pattern of test results. Our experiment was designed to test differences between stressed and control subjects and as saliva cortisol levels were still significantly enhanced in stressed subjects at the end of the test procedure, group differences in each task are caused by the stress manipulation.

A limitation of the present study is that only male subjects were included since a gender effect has been found in previous studies (e.g., Buchanan and Tranel, 2008; Jackson et al., 2006; Kelly et al., 2008; Shors et al., 2004; Stark et al., 2006). Future studies including both men and women will be required to investigate the impact of sex differences on stress effects on different memory domains (cf., Cahill, 2003; Het et al., 2005).

Our results support and extend previous findings on the complexity of effects induced by acute stress. They reinforce the importance of delineating the memory type under study when addressing stress and memory interactions (Sandi and Pinelo-Nava, 2007).

\section{ACKNOWLEDGEMENT}

The authors would like to thank Clara Rossetti and Coralie Siegmund for excellent technical assistance. This work was partially supported by intramural funding from the EPFL (to C.S.). 


\section{REFERENCES}

Abercrombie, H. C., Speck, N. S., and Monticelli, R. M. (2006). Endogenous cortisol elevations are related to memory facilitation only in individuals who are emotionally aroused. Psychoneuroendocrinology 31, 187-196.

Abrari, K., Rashidy-Pour, A., Semnanian, S., and Fathollahi, Y. (2008). Post-training administration of corticosterone enhances consolidation of contextual fear memory and hippocampal long-term potentiation in rats. Neurobiol. Learn. Mem., doi:10.1016/j.nlm.2008.10.008,

Aerni, A., Traber, R., Hock, C., Roozendaal, B., Schelling, G., Papassotiropoulos, A., Nitsch, R. M., Schnyder, U., and de Quervain, D. J. F. (2004). Low-dose cortisol for symptoms of posttraumatic stress disorder. Am. J. Psychiatry 161, 1488-1490.

Bäumler, G. (1974). Lern- und Gedächtnistest LGT-3 [Learning and Memory Test]. Göttingen, Verlag für Psychologie, Hogrefe.

Beckner, V. E., Tucker, D. M., Delville, Y., and Mohr, D. C. (2006). Stress facilitates consolidation of verbal memory for a film but does not affect retrieval. Behav. Neurosci. 120, 518-527.

Bishop, S. J. (2007). Neurocognitive mechanisms of anxiety: an integrative account. Trends $\operatorname{Cogn}$. Sci. 11, 307-316.

Buchanan, T. W., and Lovallo, W. R. (2001). Enhanced memory for emotional material following stresslevel cortisol treatment in humans. Psychoneuroendocrinology 26, 307-317.

Buchanan, T. W., and Tranel, D. (2008). Stress and emotional memory retrieval: effects of sex and cortisol response. Neurobiol. Learn. Mem. 89, 134-141.

Cahill, L. (2003). Sex-related influences on the neurobiology of emotionally influenced memory. Ann. N. Y. Acad. Sci. 985, 163-173.

Chun, M. M., and Jiang, Y. (1998). Contextual cueing: implicit learning and memory of visual context guides spatial attention. Cognit. Psychol. 36, 28-71.

Chun, M. M., and Phelps, E. A. (1999). Memory deficits for implicit contextual information in amnestic subjects with hippocampal damage. Nat. Neurosci. 2, 844-847.

Colombel, F. (2007). Memory bias and depression: a critical commentary. Encephale 33, 242-248.

Conrad, C. D., LeDoux, J. E., Magariños, A. M., and McEwen, B. S. (1999). Repeated restraint stress facilitates fear conditioning independently of causing hippocampal CA3 dendritic atrophy. Behav. Neurosci. 113 902-913.

Cordero, M.I., Kruypt, N.D., and Sandi, C. (2003a). Modulation of contextual fear conditioning by chronic stress in rats is related to individual differences in behavioral reactivity to novelty. Brain Res. 970, 242-245.

Cordero, M. I., Venero, C., Kruyt, N. D., and Sandi, C. (2003b). Prior exposure to a single stress session facilitates subsequent contextual fear conditioning. Evidence for a role of corticosterone. Horm. Behav. 44, 338-345.

Cordero, M. I., and Sandi, C. (1998). A role for brain glucocorticoid receptors in contextual fear conditioning: dependence upon training intensity. Brain Res. 786, 11-17.

Daneman, M., and Carpenter,P.A. (1980). Individual differences in working memory and reading. J. Verbal Learn. Verbal Behav. 19, 450-466.

de Kloet, E. R., Oitzl, M. S., and Joëls, M. (1999). Stress and cognition: are corticosteroids good or bad guys? Trends Neurosci. 22, 422-426.

de Quervain, D. J. (2006). Glucocorticoidinduced inhibition of memory retrieval: implications for posttraumatic stress disorder. Ann. N. Y. Acad. Sci. 1071, 216-220.

de Quervain, D. J. (2008). Glucocorticoidinduced reduction of traumatic memories: implications for the treatment of PTSD. Prog. Brain Res. 167, 239-247. deQuervain,D.J.,Aerni,A., Roozendaal, B. (2007). Preventive effect of beta-adrenoceptor blockade on glucocorticoidinduced memory retrieval deficits. Am. J. Psychiatry 164, 967-969.

de Quervain, D. J., Henke, K., Aerni, A., Treyer, V., McGaugh, J. L., Berthold, T., Nitsch, R. M., Buck,A., Roozendaal, B., and Hock, C. (2003). Glucocorticoidinduced impairment of declarative memory is associated with reduced blood flow in the medial temporal lobe. Eur. J. Neurosci. 17, 1296-1302.

de Quervain, D. J.-F., Roozendaal, B., Nitsch, R. M., McGaugh, J. L., and Hock, C. (2000). Acute cortisone administration impairs retrieval of long-term declarative memory in humans. Nat. Neurosci. 3, 313-314.

Del Arco, A., Segovia, G., Garrido, P., de Blas, M., and Mora, F. (2007). Stress, prefrontal cortex and environmental enrichment: studies on dopamine and acetylcholinase release and working memory performance in rats. Behav. Brain Res. 176, 267-273.

Ehlers, A., and Clark, D. M. (2008). Post-traumatic stress disorder: the development of effective psychological treatments. Nord. J. Psychiatry 62(Suppl. 47), 11-18.
Elzinga, B. M., and Bremner, J. D. (2002). Are the neural substrates of memory the final common pathway in posttraumatic stress disorder (PTSD)? J. Affect. Disord. 70, 1-17.

Gidron,Y.,Barak, T.,Henik,A., Gurman, G. and Stiener, O. (2002). Implicit learning of emotional information under anesthesia. Neuroreport 13, 139-142.

Greene, A. J., Gross. W. L., Elsinger, C. L., and Rao, S. M. (2007). Hippocampal differentiation without recognition: an fMRI analysis of the contextual cueing task. Learn. Mem. 14, 548-553.

Het, S., Ramlow, G., and Wolf, O.T. (2005). A meta-analytic review of the effects of acute cortisoladministration on human memory. Psychoneuroendocrinology 30, 771-784.

Jackson, E. D., Payne, J. D., Nadel, L., and Jacobs, W. J. (2006). Stress differentially modulates fear conditioning in healthy men and women. Biol. Psychiatry 59, 516-522.

Joëls, M. (2006). Corticosteroid effects in the brain: U-shape it. Trends Pharmacol. Sci. 27, 244-250.

Kelly, M.M., Tyrka, A. R., Anderson, G. M., Price, L. H., and Carpenter, L. L. (2008). Sex differences in emotional and physiological responses to the Trier Social Stress Test. J. Behav. Exp. Psychiatry 39, 87-98.

Kirschbaum, C., Pirke, K. M., and Hellhammer, D. H. (1993). The "Trier Social Stress Test" - a tool for investigating psychobiological stress responses in a laboratory setting. Neuropsychobiology 28, 76-81.

Kirschbaum, C., Wolf, O. T., Wippich, W., and Hellhammer, D. H. (1996). Stressand treatment induced cortisol levels associated with impaired declarative memory in healthy adults. Life Sci. 58 , 1475-1483.

Kuhlmann, S., Kirschbaum, C., and Wolf, O. T. (2005). Effects on oral cortisol treatment in healthy young women on memory retrieval of negative and neutral words. Neurobiol. Learn. Mem. 83, 158-162.

Lupien, S. J., Fiocco, A., Wan, N., Maheu, C. L., Schramek, T., and Tu, M. T. (2005). Stress hormones and human memory function across the lifespan. Psychoneuroendocrinology 30, 225-242.

Lupien, S. J., Gaudreau, S., Tchiteya, B. M., Maheu, F., Sharma, S., Nair, N. P. V. Hauger, R. L., McEwen, B. S., and Meaney, M. J. (1997). Stress-induced declarative memory impairment in healthy elderly subjects: relationship to cortisol reactivity. J. Clin. Endocrinol. Metab. 82, 2070-2075.

Lupien, S. J., Gillin, C. J., and Hauger, R. L. (1999). Working Memory is more sensitive than declarative memory to the acute effects of corticosteroids: a dose-response study in humans. Behav. Neurosci. 113, 420-430.

Lupien, S. J., and Lepage, M. (2001). Stress, memory, and the hippocampus: can't live with it, can't live without it. Behav. Brain Res. 127, 137-158.

Lupien, S. J., Maheu, F., Tu, M., Fiocco, A., and Schramek, T. E. (2007). The effects of stress and stress hormones on human cognition: implications for the field of brain and cognition. Brain Cogn. 65, 209-237.

Lupien, S. J., and McEwen, B. S. (1997). The acute effects of corticosteroids on cognition: integration of animal and human model studies. Brain Res. Rev. 24, 1-27.

Lupien, S. J., and Schramek, T. E. (2006). The differential effects of stress on memory consolidation and retrieval: a potential involvement of reconsolidation? Theoretical comment on Beckner et al. (2006). Behav. Neurosit. 120, 735-738.

McNally, R. J. (1997). Implicit and explicit memory for trauma-related information in PTSD. Ann. N. Y. Acad. Sci. 821, 219-224.

Meier, B. (2001). Verschwinden Dissoziationen zwischen impliziten und explizitem Gedächtnis, wenn die Reliabilität der Tests vergleichbar ist? Ein Beispiel. [Do dissociations between implicit and explicit memory disappear, when reliability of the tests is comparable? An example.] Z. Exp. Psychol. 48, 207-213.

Meier, B., Theiler-Bürgi, M., and Perrig, W. (2009). Levels of processing and amnesia affect perceptual priming in fragmented picture naming. Int. J. Neurosci. 119.

Moradi, A. R., Taghavi, R., NeshatDoost, H. T., Yule, W., and Dalgleish, T (2000). Memory bias for emotional information in children and adolescents with posttraumatic stress disorder: a preliminary study. J. Anxiety Disord. 14, 521-534.

Morris, R. G. (2006). Elements of a neurobiological theory of hippocampal function: the role of synaptic plasticity, synaptic tagging and schemas. Eur. J. Neurosci. 23, 2829-2846.

Newcomer, J. W., Craft, S., Hershey, T., Askins, K., and Bargett, M. E. (1994). Glucocorticoid-induced impairment in declarative memory performance in adult humans. J. Neurosci. 14, 2047-2053.

Newcomer, J. W., Selke, G., Melson, A. K., Hershey, T., Craft, S., Richards, K., and Alderson, A. L. (1999). Decreased memory performance in healthy humans induced by stress-level cortisol treatment. Arch. Gen. Psychiatry $56,527-533$. 
Oei, N. Y. L., Everaerd, W. T. A. M., Elzinga, B. M., van Well, S., and Bermond, B. (2006). Psychological stress impairs working memory at high loads: an association with cortisol levels and memory retrieval. Stress 9 , 133-141.

Olson, M. A., and Fazio, R. H. (2001). Implicit attitude formation through classical conditioning. Psychol. Sci. 12, 413-417.

Payne, J. D., Jackson, E. D., Hoscheidt, S., Ryan, L., Jabos, W. J., and Nadel, L. (2007). Stress administered prior to encoding impairs neutral but enhances emotional long-term episodic memories. Learn. Mem. 14, 861-868.

Putman, P., van Honk, J., Kessels, R. P. C., Mulder, M., and Koppeschaar, H. P. F. (2004). Salivary cortisol and short and long-term memory for emotional faces in healthy young women. Psychoneuroendocrinology 29, 953-960.

Rinck, M., and Becker, E. S. (2005). A comparison of attentional biases and memory biases in women with social phobia and major depression. J. Abnorm. Psychol. 114, 62-74.

Robinson, S. J., Sünram-Lea, S. I., Leach, J., and Owen-Lynch, P. J. (2008). The effects of exposure to an acute naturalistic stressor on working memory, state anxiety and salivary cortisol concentrations. Stress 11, 115-124.

Roozendaal, B. (2002). Stress and memory: opposing effects of glucocorticoids on memory consolidation and memory retrieval. Neurobiol. Learn. Mem. 78, 578-595.

Roozendaal, B., Okuda, S., de Quervain, D. J., and McGaugh, J. L. (2006). Glucocorticoids interact with emotion-induced noradrenergic activation in influencing different memory functions. Neursocience 138, 901-910.

Roozendaal, B., Quirarte, G. L., and McGaugh, J.L. (2002). Glucocorticoids interact with the basolateral amygdala beta-adrenoceptor-cAMP/cAMP/ PKA system in influencing memory consolidation. Eur. J. Neurosci. 15, 553-560.

Rubin, D. C., Berntsen, D., and Bohni, M. K. (2008). A memory- based model of posttraumatic stress disorder: evaluating basic assumptions underlying the PTSD diagnosis. Psychol. Rev. 115, 985-1011.

Sandi, C., Loscertales, M., and Guaza, C. (1997). Experience-dependent facilitating effect of corticosterone on spatial memory formation in the water maze. Eur. J. Neurosci. 9, 637-642.

Sandi, C., Merino, J. J., Cordero, M. I., Touyarot, K., and Venero, C. (2001). Effects of chronic stress on contextual fear conditioning and the hippocampal expression of the neural cell adhesion molecule, its polysialylation, and L1. Neuroscience 102, 329-339.

Sandi, C., and Pinelo-Nava, M. T. (2007). Stress and memory: behavioral effects and neurobiological mechanisms. Neural Plast. 2007, 78970.

Sandi, C., and Rose, S. P. (1997). Trainingdependent biphasic effects of corticosterone in memory formation for a passive avoidance task in chicks. Psychopharmacology 133, 152-160.

Sauro, M. D., Jorgensen, R. S., and Pedlow, C. T. (2003). Stress, glucocorticoids and memory: a meta-analytic review. Stress 6, 235-245.

Schelling, G. (2008). Post-traumatic stress disorder in somatic disease: lessons from critically ill patients. Prog. Brain Res. 167, 229-237.

Schelling, G., Kilger, E., Roozendaal, B., de Quervain, D.J. F., Briegel, J., Dagge, A., Rothenhäusler, H. B., Krauseneck, T., Nollert, G., and Kapfhammer, H. P. (2004). Stress doses of hydrocortisone, traumatic memories, and symptoms of posttraumatic stress disorder in patients after cardiac surgery: a randomized study. Biol. Psychiatry 55, 627-633.

Schoofs, D., Preuss., D., and Wolf, O. T. (2008). Psychosocial stress induces working memory impairments in an n-back paradigm. Psychoneuroendocrinology 33, 643-653.

Selden, N. R., Cole, B. J., Everitt, B. J., and Robbins, T. W. (1990). Damage to ceruleo-cortical noradrenergic projections impairs locally cued but enhances spatially cued water maze acquisition. Behav. Brain Res. 39, 29-51.
Shors, T.J. (2001).Acute stress rapidly and persistently enhances memory formation in the male rat. Neurobiol. Learn. Mem. 75, 10-29.

Shors, T.J. (2004). Learning during stressful times. Learn. Mem. 11, 137-144.

Shors, T. J. (2006). Stressful experience and learning across the lifespan. Аnпu. Rev. Psychol. 57, 55-85.

Shors, T. J., and Beylin, A. V. (2003). Glucocorticoids are necessary for enhancing the acquisition of associative memories after acute stressful experience. Horm. Behav. 43, 124-131.

Shors, T. J., Falduto, J., and Leuner, B. (2004). The opposite effects of stress on dendritic spines in male vs. female rats are NMDA receptor-dependent. Eur. J. Neurosci. 19, 145-150.

Smeets, T., Otgaar, H., Candel, I., and Wolf, O. T. (2008). True of false? Memory is differentially affected by stress-induced cortisol elevations and sympathetic activity at consolidation and retrieval. Psychoneuroendocrinology 33, 1378-1386.

Snodgrass, J. G., and Vanderwart, M. (1980). A standardized set of 260 pictures: norms for name agreement, image agreement, familiarity, and visual complexity. J. Exp. Psychol. [Hum Learn.] 6, 174-215.

Soravia, L. M., Heinrichs, M., Aerni, A., Maroni, C., Schelling, G., Ehlert, U., Roozendaal, B., and de Quervain, D. J. (2006). Glucocorticoids reduce phobic fear in humans. PNAS 103, 5585-5590.

Stark, R., Wolf, O. T., Tabbert, K., Kagerer, S., Zimmermann, M., Kirsch, P., Schienle, A., and Vaitl, D. (2006). Influence of the stress hormone cortisol on fear conditioning in humans: evidence for sex differences in the response of the prefrontal cortex. Neuroimage 32, 1290-1298.

Tops, M., van der Pompe, G., Baas, D., Mulder, L. J. M., Den Boer, J. A., Meijman, T. F., and Korf, J. (2003). Acute cortisol effects on immediate free recall and recognition of nouns depend on stimulus valence. Psychophysiology 40, 167-173.
Watkins, P. C., Martin, C. K., and Stern, L. D. (2000). Unconscious memory bias in depression: perceptual and conceptual processes. J. Abnorm. Psychol. 109, 282-289.

Weis, F., Kilger, E., Roozendaal, B., de Quervain,D.J.F.,Lamm,P.,Schmidt, M., Schmölz,M., Briegel, J., and Schelling, G. (2006). Stress doses of hydrocortisone reduce chronic stress symptoms and improve health-related quality of life in high-risk patients after cardiac surgery: a randomized study. J. Thorac. Cardiovasc. Surg. 131, 277-282.

Wolf, O. T. (2003). HPA axis and memory. Best Pract. Res. Clin. Endocrinol. Metab. 17, 287-299.

Wolf, O. T. (2006). Effects of stress hormones on the structure and function of the human brain. Expert Rev. Endocrinol. Metab. 1, 623-632.

Wolf, O. T. (2008). The influence of stress hormones on emotional memory: relevance for psychopathology. Acta Psychol. 127, 513-531.

Zorawski, M., Blanding, N. Q., Kuhn, C. M., and LaBar, K. S. (2006) Effects of stress and sex on acquisition and consolidation of human fear conditioning. Learn. Mem. 13, 441-450.

Conflict of Interest Statement: The authors declare that the research was conducted in the absence of any commercial or financial relationships that could be construed as a potential conflict of interest.

Received: 22 October 2008; paper pending published: 30 November 2008; accepted: 28 December 2008; published online: 15 January 2009.

Citation: Luethi M, Meier B and Sandi C (2009) Stress effects on working memory, explicit memory, and implicit memory for neutral and emotional stimuli in healthy men. Front. Behav. Neurosci. (2009) 2:5. doi: 10.3389/neuro.08.005.2008

Copyright $\odot 2009$ Luethi, Meier and Sandi. This is an open-access article subject to an exclusive license agreement between the authors and the Frontiers Research Foundation, which permits unrestricted use, distribution, and reproduction in any medium, provided the original authors and source are credited. 